\title{
High energy parton-parton amplitudes from lattice QCD and the stochastic vacuum model
}

\author{
A. F. Martini, M. J. Menon and D. S. Thober \\ Instituto de Física Gleb Wataghin, \\ Universidade Estadual de Campinas, Unicamp, \\ 13083-970 Campinas, SP, Brazil
}

\begin{abstract}
Making use of the gluon gauge-invariant two-point correlation function, recently determined by numerical simulation on the lattice in the quenched approximation and the stochastic vacuum model, we calculate the elementary (parton-parton) amplitudes in both impact-parameter and momentum transfer spaces. The results are compared with those obtained from the Krämer and Dosch ansatz for the correlators. Our main conclusion is that the divergences in the correlations functions suggested by the lattice calculations do not affect substantially the elementary amplitudes. Phenomenological and semiempirical information presently available on elementary amplitudes is also referred to and is critically discussed in connection with some theoretical issues.
\end{abstract}

13.85.Dz, 12.38.Gc, 12.38.Lg

\section{INTRODUCTION}

The theoretical investigation of quark-quark scattering is one of the topical problems in high-energy hadron physics. In principle, the possibility to predict this amplitude directly from quantum chromodynamics (QCD) could lead to important insight concerning high-energy soft processes and meanly elastic hadron scattering. Although some phenomenological and semiempirical information already exists [1,2], the determination of this amplitude from a pure QCD approach (model independent) is still an open problem. However, recently, remarkable progress has been achieved, starting with the nonperturbative approach by Landshoff and Nachtmann [3], in which quarks are coupled to Abelian gluons (gluon condensate). In a non-Abelian model, the amplitudes of quarks at high energies were computed by Nachtmann through the use of the eikonal method [4. Analogous version was then developed by Krämer and Dosch 5 in the context of the stochastic vacuum model (SVM) 6., (7). This model describes the low frequencies contributions in the functional integral of QCD in terms of a stochastic process by means of a cluster expansion [8] and gives a good description of the heavy quark potential [9]. The most general form of the lowest cluster is the gauge invariant two-point field strength correlator [6, 7, 10]

$$
\begin{aligned}
& \left\langle\mathbf{F}_{\mu \nu}^{C}(x) \mathbf{F}_{\rho \sigma}^{D}(y)\right\rangle \\
& =\delta^{C D} g^{2} \frac{\langle F F\rangle}{12\left(N_{c}^{2}-1\right)}\left(\left(\delta_{\mu \rho} \delta_{\nu \sigma}-\delta_{\mu \sigma} \delta_{\nu \rho}\right) \kappa D\left(z^{2} / a^{2}\right)\right. \\
& +\frac{1}{2}\left[\partial_{\mu}\left(z_{\rho} \delta_{\nu \sigma}-z_{\sigma} \delta_{\nu \rho}\right)+\partial_{\nu}\left(z_{\sigma} \delta_{\mu \rho}-z_{\rho} \delta_{\mu \sigma}\right)\right] \\
& \left.\times(1-\kappa) D_{1}\left(z^{2} / a^{2}\right)\right),
\end{aligned}
$$

where $z=x-y$ is the two-point distance, $a$ is a characteristic correlation length, $\kappa$ a constant, $g^{2}\langle F F\rangle$ the gluon condensate, and $N_{c}$ the number of colors, $C, D=$ $1, \ldots, N_{c}^{2}-1$. The two scalar functions $D$ and $D_{1}$ describe the correlations and are normalized as $D(0)=D_{1}(0)=1$.

These correlation functions play a central role in the application of the SVM to high-energy scattering. Once one has information about $D$ and $D_{1}$, the SVM leads to the determination of the elementary quark-quark scattering amplitude and this is the point we are interested in.

Numerical determinations of these correlation functions, in limited interval of physical distances, exist from lattice QCD in the quenched approximation. The first determination, by Di Giacomo and Panagopoulos, covered the interval 0.4 to $1.0 \mathrm{fm}$ [11. Recently, through improved technique (larger lattice) the range was extended down to $0.1 \mathrm{fm}$ by Di Giacomo, Meggiolaro, and Panagopoulos [12] and this brought new and important insights on the subject, as will be discussed.

On the other hand, Krämer and Dosch introduced a suitable ansatz for the correlation function $D$ and $D_{1}$ [5], which is in agreement with the early lattice results in the range 0.4-1.0 fm 10]. Using the SVM they acchieved good descriptions of the experimental data on total cross sections and slopes of the elastic amplitudes in hadronic processes [5, 10]. Also, with the same ansatz, Grandel and Weise calculated differential cross sections through the eikonal approximation (multiple diffraction Glauber theory). Introducting a monopole parametrization for the form factors, a satisfactory description of experimental data on $p p$ and $\bar{p} p$ elastic scattering at small momentum transfer was obtained [13].

In this paper we make use of the recent lattice results for the correlation functions and, through the SVM, we calculate the gauge invariant elementary amplitudes in both impact-parameter and momentum transfer spaces. The results are then compared with those obtained through the Krämer-Dosch ansatz and also with some phenomenological and semiempirical information avail- 
able. Since in the SVM the amplitudes are characterized by scattering amplitudes for Wilson loops in Minkowski space, they will generically be referred to as elementary parton-parton amplitudes.

The material is organized as follows. In Sec. II we briefly recall the essential formulas of the SVM connecting the field strength correlator (11) with the partonparton amplitudes and in Sec. III we review the correlation functions from lattice calculations and the ansatz by Krämer and Dosch. With this information in Sec. IV we present our calculations and results in some detail. Discussions and critical remarks concerning nonperturbative QCD, phenomenological, and semiempirical results on parton-parton amplitudes are presented in Sec. \ and conclusions in Sec. VI.

\section{PARTON-PARTON AMPLITUDES IN THE STOCHASTIC VACUUM MODEL}

In the nonperturbative QCD framework referred to in the last section, the study of the elementary scattering is based on the amplitudes of quarks moving on lightlike paths in an external field. In the Nachtmann approach the quarks involved in a scattering pick up an eikonal phase in traveling through the nonperturbative QCD vacuum. In order to have gauge invariant Dirac wave function solutions a Wilson loop is proposed to represent each quark. In this context the no-color exchange parton-parton (loop-loop) amplitude can be written as 44

$$
\begin{aligned}
\gamma & =\left\langle\operatorname{Tr}\left[\mathcal{P} e^{-i g \int_{\text {loop } 1} d \sigma_{\mu \nu} F_{\mu \nu}(x ; w)}-1\right]\right. \\
& \left.\times \operatorname{Tr}\left[\mathcal{P} e^{-i g \int_{\text {loop } 2} d \sigma_{\rho \sigma} F_{\rho \sigma}(y ; w)}-1\right]\right\rangle
\end{aligned}
$$

where \langle\rangle means the functional integration over the gluon fields, the integrations are over the respective loop areas, and $w$ is a common reference point from which the integrations are performed.

This expression is simplified in the Krämer and Dosch description by taking the Wilson loops on the light-cone. In the SVM the leading order contribution to the amplitude is given by [5]

$$
\gamma(b)=\eta \epsilon^{2}(b)
$$

where $b$ is the impact parameter, $\eta$ is a constant depending on normalizations [see Eqs. 22) and (24)], and

$$
\epsilon(b)=g^{2} \iint d \sigma_{\mu \nu} d \sigma_{\rho \sigma} \operatorname{Tr}\left\langle F_{\mu \nu}(x ; w) F_{\rho \sigma}(y ; w)\right\rangle .
$$

Here $\left\langle g^{2} F_{\mu \nu}(x ; w) F_{\rho \sigma}(y ; w)\right\rangle$ is the Minkowski version of the gluon correlator. We will return to this point later.

After a two-dimensional integration, $\epsilon(b)$ may be expressed in terms of the correlation functions in Eq. (11) by [5]

$$
\begin{array}{r}
\epsilon(b)=\epsilon_{\mathrm{I}}(b)+\epsilon_{\mathrm{II}}(b), \\
\epsilon_{\mathrm{I}}(b)=\kappa\left\langle g^{2} F F\right\rangle \int_{b}^{\infty} d b^{\prime}\left(b^{\prime}-b\right) \mathcal{F}_{2}^{-1}\left[D\left(-q^{2}\right)\right]\left(b^{\prime}\right) \\
\epsilon_{\mathrm{II}}(b)=(1-\kappa)\left\langle g^{2} F F\right\rangle \mathcal{F}_{2}^{-1}\left[\frac{d}{d q^{2}} D_{1}\left(-q^{2}\right)\right](b),
\end{array}
$$

where $q^{2}$ is the four-momentum transfer squared and $\mathcal{D}=$ $D$ or $D_{1}$,

$$
\mathcal{D}\left(k^{2}\right)=\mathcal{F}_{4}\left[\mathcal{D}\left(z^{2}\right)\right]
$$

with $\mathcal{F}_{n}$ denoting a $n$-dimensional Fourier transform.

In the impact parameter space $\gamma$ represents the elementary profile function, from which the elementary partonparton scattering amplitude is calculated through a twodimensional Fourier transform:

$$
f\left(q^{2}\right)=\int_{0}^{\infty} b d b J_{0}(q b) \gamma(b)
$$

where $J_{0}$ is the Bessel function.

With the above formalism, once one has inputs for the correlation functions $D(z)$ and $D_{1}(z)$ the elementary amplitudes in the impact parameter and transfer momentum spaces, Eqs. (3) and (9), respectively, may, in principle, be evaluated through Eqs. (5)-(8).

\section{CORRELATION FUNCTIONS}

We now recall the theoretical information available about the correlation functions $D$ and $D_{1}$, namely, that obtained from lattice QCD and the ansatz introduced by Krämer and Dosch. In Sec. IV we use these functions in order to calculate the elementary amplitudes. The translation from Euclidean to Minkowski space will be discussed in the next section.

\section{A. Lattice results}

The first determination of the correlation functions from lattice QCD in the quenched approximation was obtained through the cooling technique 14,15, with a lattice of size $16^{4}$ and in the interval of physical distance 0.4 to $1.0 \mathrm{fm}$ [11. In this range the theoretical data showed agreement with an exponential decrease of both correlators with the distance. In particular, using the notation of Ref. [10], the function $D$ is parametrized by

$$
\kappa\left\langle g^{2} F F\right\rangle D\left(-\frac{r^{2}}{a^{2}}\right)=24 C \exp \left(-\frac{r}{\lambda}\right),
$$

where $r$ is the physical distance (Euclidean space), and

$$
\lambda=\frac{1}{183 \Lambda}, \quad \frac{C}{\Lambda^{4}}=3.6 \times 10^{8},
$$


with a typical statistical error of a few percent. In Ref. 111 the value of $\Lambda$ was determined from string tension studies [16] leading to

$$
C=137 \mathrm{fm}^{-4}, \quad \lambda=0.22 \mathrm{fm},
$$

which corresponds to $\Lambda=4.9 \mathrm{MeV}$.

Recently new results were obtained on a $32^{4}$ lattice allowing the determination of the correlators at distances down to $0.1 \mathrm{fm} \mathrm{[12].} \mathrm{A} \mathrm{novel} \mathrm{result} \mathrm{was} \mathrm{the} \mathrm{appearance}$ of a deviation from the exponential behavior at shorter distances $(<0.4 \mathrm{fm})$, indicating a $1 / x^{4}$ divergence at the origin. Putting together the data of Refs. [11] and [12], good agreement is obtained within statistical errors, with a parametrization of the form 12 .

$$
\begin{aligned}
& \kappa\left\langle g^{2} F F\right\rangle D\left(z^{2}\right) \\
& =24\left[A \exp \left(-\frac{|z|}{\lambda_{A}}\right)+\frac{B}{|z|^{4}} \exp \left(-\frac{|z|}{\lambda_{B}}\right)\right], \\
& (1-\kappa)\left\langle g^{2} F F\right\rangle D_{1} \\
& =24\left[A_{1} \exp \left(-\frac{|z|}{\lambda_{A}}\right)+\frac{B_{1}}{|z|^{4}} \exp \left(-\frac{|z|}{\lambda_{B}}\right)\right] .
\end{aligned}
$$

As before, from string tension information on $\Lambda, \lambda_{A}=$ $0.22 \mathrm{fm}$ and $\lambda_{B}=0.43 \mathrm{fm}$. The typical error is again of the order of a few percent. In Sec. [IV] we test and discuss the influence of the divergent terms.

\section{B. Krämer-Dosch ansatz}

In the approach of Ref. [10, the effect of $D_{1}\left(z^{2} / a^{2}\right)$ is neglected since, from QCD lattice calculation, the value of $\kappa$ in Eq. (11) is $\approx 1$ [11]. So, we will consider only $D$ for this case.

Assuming functions which have a well defined Fourier transform and that can be analitically continuated to Euclidean world in the momentum space, Krämer and Dosch introduced a family of functions depending on an integer parameter $n$ [5]. These functions may be analitically integrated and the selection of the $n$ value was made by comparison with the early lattice results, Eq. (10). In the Euclidean space the fit in the interval $0.5-0.8 \mathrm{fm}$ (early lattice results) led to $n=4$ and for $\Lambda=4.4 \mathrm{MeV}$ it was obtained $a=0.35 \mathrm{fm}$ and $\kappa\left\langle g^{2} F F\right\rangle=1.774 \mathrm{GeV}^{4}$ [10]. The final result for the correlation function with $n=4$ is given by 10 ]

$$
D_{\mathrm{KD}}^{(4)}(x)=x\left[K_{1}(x)-\frac{x}{4} K_{0}(x)\right],
$$

where $K_{1}, K_{0}$ are Bessel functions and $x$ is a dimensionless variable

$$
x=\frac{3 \pi}{8} \frac{z}{a} .
$$

\section{Remarks on the correlation functions}

In what follows, it is important to stress that the Krämer-Dosch ansatz for the correlation function $D$ is an analytical form, firstly selected by analytical continuation and asymptotic limit conditions and then through fit to early lattice information, Eq. (10). On the other hand, "real" lattice results correspond to sets of discret theoretical points with errors, in a finite interval of physical distances $(0.1-1.0 \mathrm{fm})$. The parametrizations introduced in both early 111 and recent 12 results, extrapolate this interval down and above. Although, presently, there is no theoretical informations on these extrapolated regions we will assume the parametrizations (13) and (14) as representing lattice results and will refer as lattice parametrizations. We will return to this point in Secs. IV A and V C.

The results reviewed in this section for the correlation function $D$ are displayed in Fig. 1 for comparison and this deserves some discussion. We plotted the curves corresponding to the early lattice result, Eqs. (10) and (12), the new lattice result, Eq. (13), and the Kramer-Dosch ansatz, Eq. (15). As mentioned above, the final form of this ansatz was obtained through a fit to the early lattice parametrization in the interval $0.4-0.8 \mathrm{fm}$. From Fig. 11, there is a discrepancy between these two curves in the above interval, which does not appear in Fig. 10 of Ref. [10]. The reason is the different values of the parameter $\Lambda$, namely, $4.4 \mathrm{MeV}$ in Ref. 10 and $4.9 \mathrm{MeV}$ in Ref. 111 and Fig. 11. This difference is significant since, for example, the parameter $C$ in Eq. (11) is proportional to $\Lambda^{4}$. On the other hand, the differences between early and new lattice results in the above interval are compatible with the errors associated with each curve.

\section{PARTON-PARTON AMPLITUDES}

In the last section we reviewed the theoretical information presently available concerning the correlation functions, namely, the recent lattice parametrizations, Eqs. (13) and (14), and the result from the ansatz by Krämer and Dosch, Eq. (15).

In the context of the SVM (Sec. III), the corresponding predictions for the parton-parton amplitudes are obtained, in principle, through four steps, envolving the following calculations: (1) $D(k)$ and $D_{1}(k)$ by the four-dimensional Fourier transform, Eq. (8); (2) two-dimensional inverse Fourier transforms of $D(k)$ and $d D_{1} / d k^{2}$, which enter in Eqs. (6) and (7); (3) eikonal phase through Eqs. (6) and (7); (4) elementary amplitudes in the impact parameter space (profile function), Eq. (3), and in the transfer momentum space (scattering amplitude), Eq. (9). These are the central points of this work and in this section we present and discuss the calculations in some detail. 


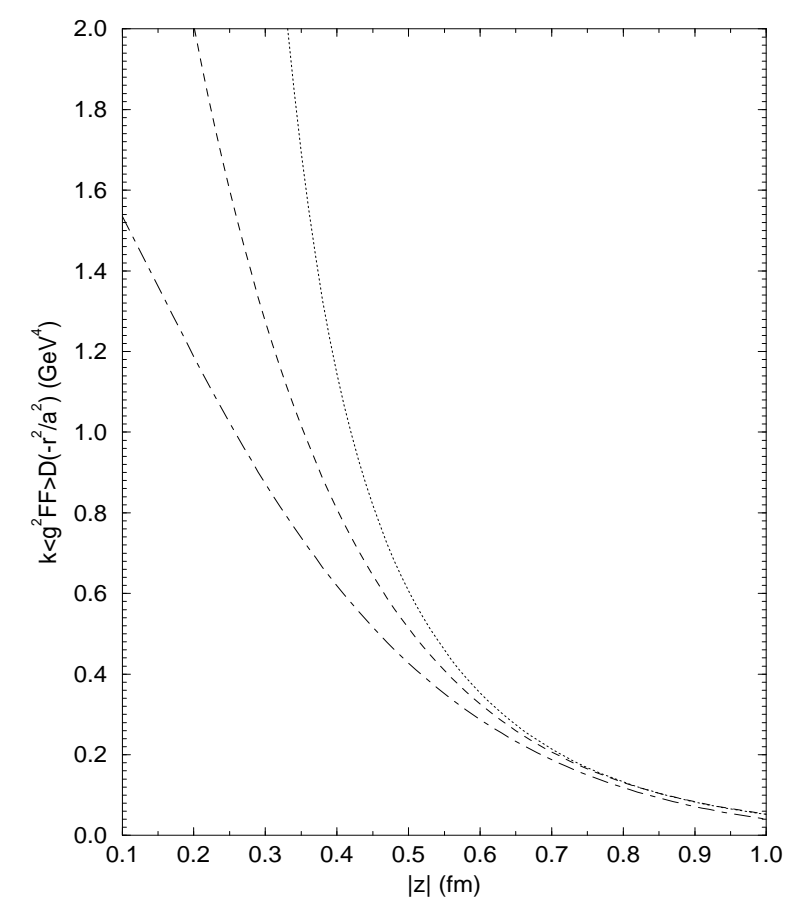

FIG. 1. Correlation function $D$ given by (a) recent lattice parametrization, Eq. 13 (dotted); (b) early lattice parametrization, Eq. (10) (dashed); (c) Krämer and Dosch ansatz, Eq. (15) (dot-dashed).

\section{A. Results from lattice parametrizations}

Our basic assumptions in using lattice results are the following.

(a) We consider the parametrizations (13) and (14) as representing the correlation functions and so, the correlation lenght has the value $a=0.22 \mathrm{fm}$.

(b) The effect of the extrapolation down to $0.1 \mathrm{fm}$ will be investigated by taking account or not of the divergent term $1 /|z|^{4}$, that is, taking or not $B=0$ and $B_{1}=0$ in Eqs. (13) and (14), respectivelly.

(c) We consider the lattice parametrizations, Eqs. (13) and (14), as representing the correlator functions in the Euclidean world. With this assumption the above correlation functions enter into Eq. (1), with the adequated tensor structures, and then directly in Eq. (8).

In using the above assumptions we seek to see the consequences in terms of the elementary amplitudes.

The first step referred to before concerns the fourdimensional transform

$$
\mathcal{D}(k)=F_{4}[\mathcal{D}(z)]=\int d^{4} z \mathcal{D}(z) \exp (i \mathbf{k} \cdot \mathbf{z})
$$

for $\mathcal{D}=D, D_{1}$, here in Euclidean space according to assumption (c) above. Although the lattice data are limited to the interval $0.1-1.0 \mathrm{fm}$, the parametrizations (13) and (14) extend to all the space and include the divergent term $|z|^{-4}$. However, we found that when the lower integral limit $z_{m}$ becomes smaller than $\approx 10^{-3} \mathrm{fm}$, numeri- cal evaluation of this transform [17, for both correlators, may be put in the form

$$
\mathcal{D}(k)=\hat{d}(k)+C\left(z_{m}\right)
$$

where $\hat{d}(k)=d_{1}(k), d(k)$ are smooth (finite) decreasing functions of $k$, and $C\left(z_{m}\right)$ is a constant which increases when the lower integral limit becomes smaller. This effect is shown in Fig. 22.
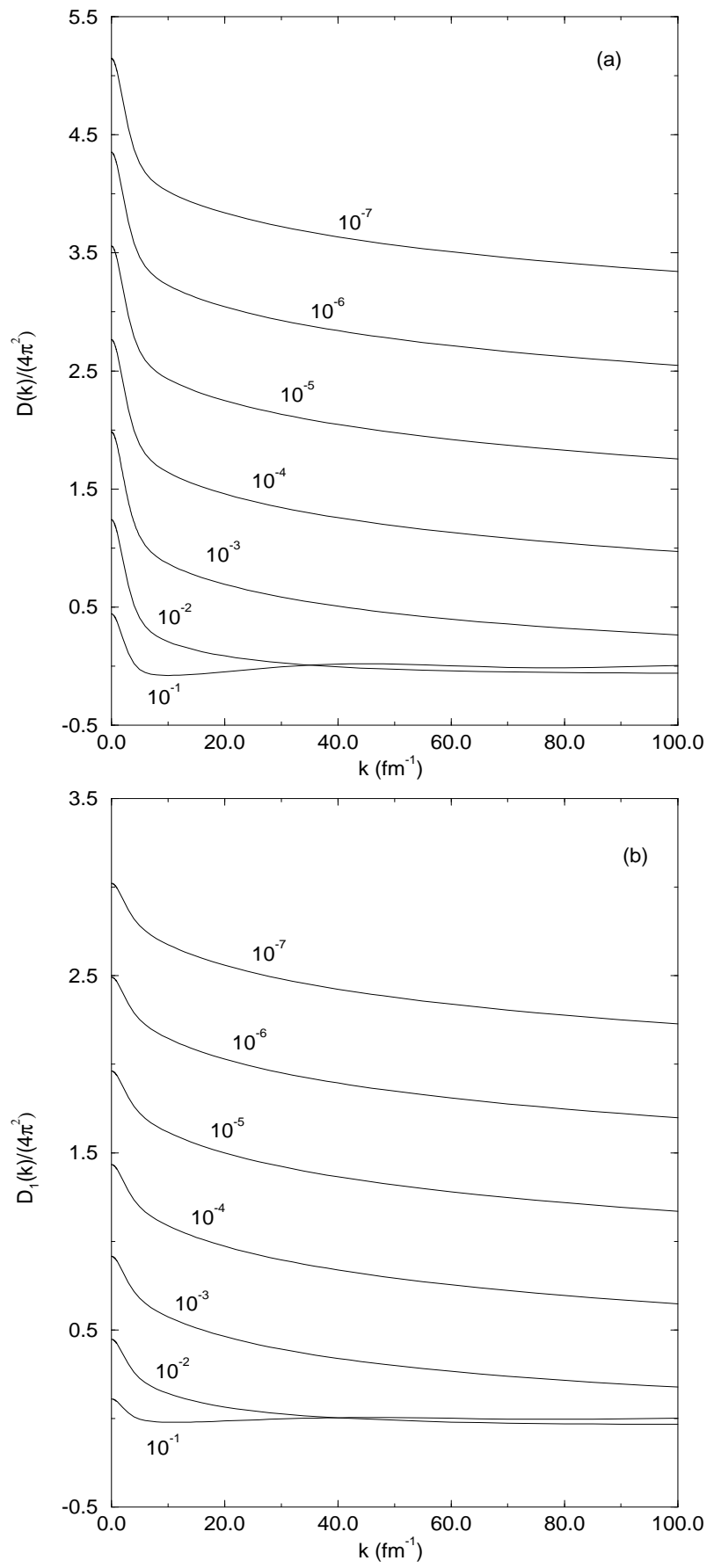

FIG. 2. Four-dimensional Fourier transform of $D(z)$, Eq. (16), and the effect of the lower integral limit $z_{m}$ (between $10^{-1}$ and $10^{-7}$ ) as represented by Eq. (17): (a) $\hat{d}(k)=d(k)$; (b) $\hat{d}(k)=d_{1}(k)$. 
Now, in the second step, we should calculate the two-dimensional Fourier transforms of $D(k)$ and $d D_{1}\left(k^{2}\right) / d k^{2}$. Because of the derivative in the last case the constant $C\left(z_{m}\right)$ may be neglected. In the former case, since the Fourier transform of $C\left(z_{m}\right)$ leads to a $\delta$ function, its effect in the calculation of the eikonal phase $\epsilon_{I}$ in Eq. (5) has also no influence.

With this, carring out the numerical integration down to $10^{-3} \mathrm{fm}$ we have a stable behavior for $\hat{d}(k)$ in both cases (see Fig. 2). The numerical results for $d(k)$ and $d_{1}(k)$ are shown in Fig. 3. We then proceed to fit these points through the CERN-MINUIT routine 18] by functions of the type

$$
\begin{array}{r}
d(k)=\sum_{j=1}^{2} a_{j} \exp \left(-b_{j} k\right)+a_{3} \exp \left(-b_{3} k^{2}\right), \\
d_{1}(k)=\sum_{j=1}^{2} a_{1 j} \exp \left(-b_{1 j} k\right)+a_{13} \exp \left(-b_{13} k^{2}\right),
\end{array}
$$

also shown in Fig. 3. The values of the free parameters

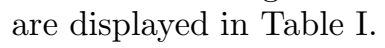

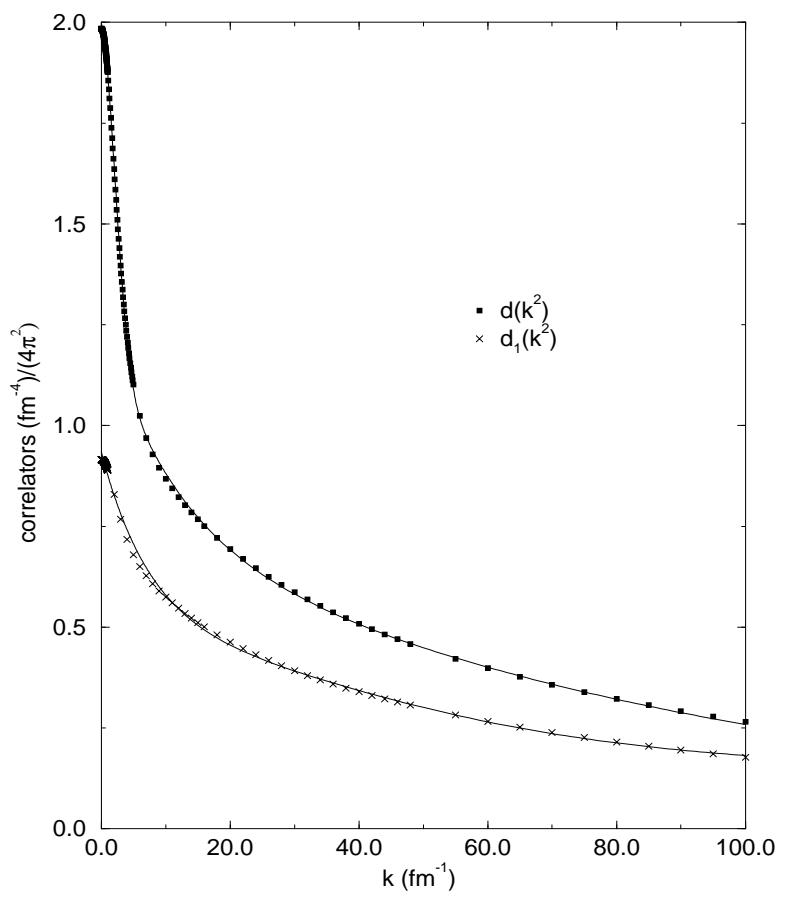

FIG. 3. Finite part of the correlation functions in momentum space, Eq. (17), calculated numerically (points) and fits through Eq. (18) (solid curves).
TABLE I. Values of the free parameters for $d(k)$ and $d_{1}(k)$ in Eq. (18). Parameters $b_{1}, b_{2}$ and $b_{11}, b_{12}$ are in fm while $b_{3}, b_{13}$ in $\mathrm{fm}^{2}$.

\begin{tabular}{ccccc}
\hline \hline $\mathrm{j}$ & $a_{j} /\left(4 \pi^{2}\right)$ & $b_{j}$ & $a_{1 j} /\left(4 \pi^{2}\right)$ & $b_{1 j}$ \\
\hline 1 & 0.50003 & 0.094168 & 0.46314 & 0.12874 \\
2 & 0.76546 & 0.010859 & 0.28780 & $0.48787 \times 10^{-2}$ \\
3 & 0.73263 & 0.104490 & 0.18440 & $0.36088 \times 10^{-3}$ \\
\hline \hline
\end{tabular}

With Eq. (18) and the derivative of $d_{1}(k)$, step 2 is performed analytically leading to 1

$$
\begin{gathered}
\mathcal{F}_{2}^{-1}\left[d\left(-q^{2}\right)\right](b)=\sum_{j=1}^{2} \alpha_{j}\left[\beta_{j} b^{2}+1\right]^{-3 / 2}+\alpha_{3} \exp \left(-\beta_{3} b^{2}\right) \\
\mathcal{F}_{2}^{-1}\left[\frac{d}{d q^{2}} d_{1}\left(-q^{2}\right)\right](b)= \\
\sum_{j=1}^{2} \alpha_{1 j}\left[\beta_{1 j} b^{2}+1\right]^{-1 / 2}+\alpha_{13} \\
\\
\times \exp \left(-\beta_{13} b^{2}\right) .
\end{gathered}
$$

From Eq. (19), in step 3, the contribution $\epsilon_{\mathrm{I}}(b)$ to the eikonal phase is analytically calculated:

$$
\begin{aligned}
\epsilon_{\mathrm{I}}(b)= & \zeta_{1} \exp \left(-\xi_{1} b^{2}\right)+\zeta_{2}\left[\operatorname{Erfc}\left(\xi_{2} b\right)\right] b+\sum_{j=1}^{2} a_{j}\left(\left[\left(\varepsilon_{j} b\right)^{2}\right.\right. \\
& \left.+1]^{1 / 2}+\varepsilon_{j} b\right)
\end{aligned}
$$

where Erfc is the complementary Error function. The contribution $\epsilon_{\mathrm{II}}(b)$, Eq. (可), is given directly by Eq. (20). Figure 4 shows both $\epsilon_{\mathrm{I}}$ and $\epsilon_{\mathrm{II}}$ as function of the impact parameter. We will discuss these results in Sec. V.

At last, step 4 is performed with $\epsilon(b)=\left[\epsilon_{\mathrm{I}}(b)+\epsilon_{\mathrm{II}}(b)\right]$ into Eq. (5), leading to the elementary profile $\gamma(b)$, Eq. (3), with normalization constant

$$
\eta=\frac{4}{9 \times 8^{2}} .
$$

Then numerical integration furnishes the corresponding elementary parton-parton amplitude $f(q)$, Eq. (9). The results for $\gamma(b)$ and $f(q)$ are shown in Figs. 5 and 6 , respectively.

As expressed in the beginning of this section, in order to investigate the effect of the divergent term in the recent lattice results, we also calculated $\gamma(b)$ and $f(q)$ neglecting these terms in both correlators, i.e., by taking account only of the first term in Eq. (13) and (14). All these results (with and without the divergent terms) are displayed in Figs. . 5 and 6 for $\gamma(b)$ and $f(q)$, respectively. We will return to this point in Sec. V, after the discussion of $\gamma(b)$ and $f(q)$ predicted from the Krämer and Dosch ansatz.

\footnotetext{
${ }^{1}$ For short, in what follows, we will refer only to the analytical structure of the parametrized and calculated functions.
} 


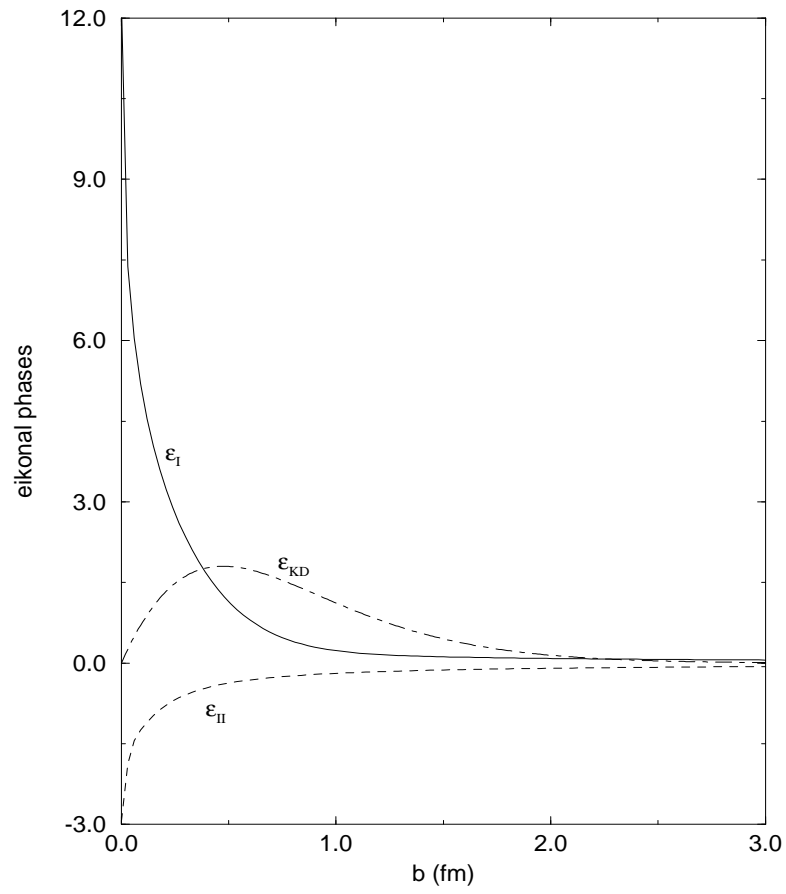

FIG. 4. Contributions for the eikonal phase, Eqs. (3), (6), and (7), from lattice ( $\epsilon_{\mathrm{I}}$ and $\epsilon_{\mathrm{II}}$ from $D$ and $D_{1}$, respectively) and Krämer-Dosch ansatz $\left(\epsilon_{\mathrm{KD}}\right)$.

\section{B. Results from Krämer-Dosch correlators}

Concerning step two, a suitable parametrization for the two-dimensional inverse Fourier transform of $D(k)$ was introduced in Ref. [10], so that step three may be performed analytically leading to

$$
\epsilon_{\mathrm{KD}}=\mathcal{K} \exp (-x) \sum_{n=0}^{4} \varrho_{n} x^{n},
$$

where $\mathcal{K}=\kappa\left\langle q^{2} F F\right\rangle a^{4} 2^{14} /\left(3^{4} \pi^{3}\right)$. This result is displayed in Fig. 1 together with those obtained through the recent lattice results.

At last, as before, the elementary profile is calculated through Eq. (3) now with normalization constant

$$
\eta=\frac{1}{9(8 \times 12)^{2}} .
$$

By numerical integration [17] we obtain the scattering amplitude. The results are shown in Figs. 5 and 6 .

\section{DISCUSSION}

In this section we first sketch the conclusions coming directly from our calculations and then present discussions related to some phenomenological/semiempirical informations available on elementary amplitudes and also some theoretical issues.
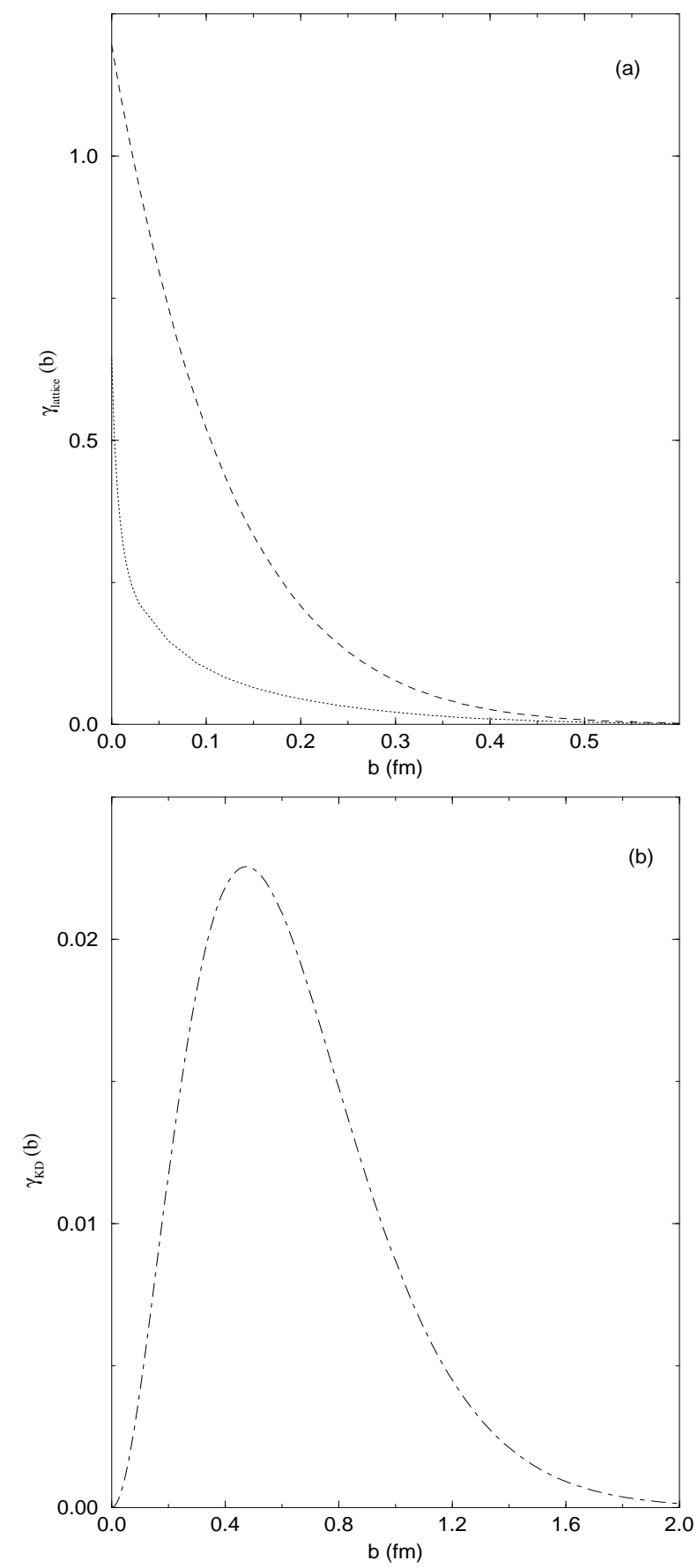

FIG. 5. Elementary (parton-parton) profile functions, Eq. (3): (a) from lattice parametrizations with the divergent term (dotted), without the divergent term (dashed); (b) from the Krämer-Dosch ansatz.

\section{A. Partial conclusions}

The main results of our calculations are displayed in Figs. 4, 局 and 6 (corresponding to eikonals, profiles, and amplitudes, respectively) and lead to the following conclusions. 


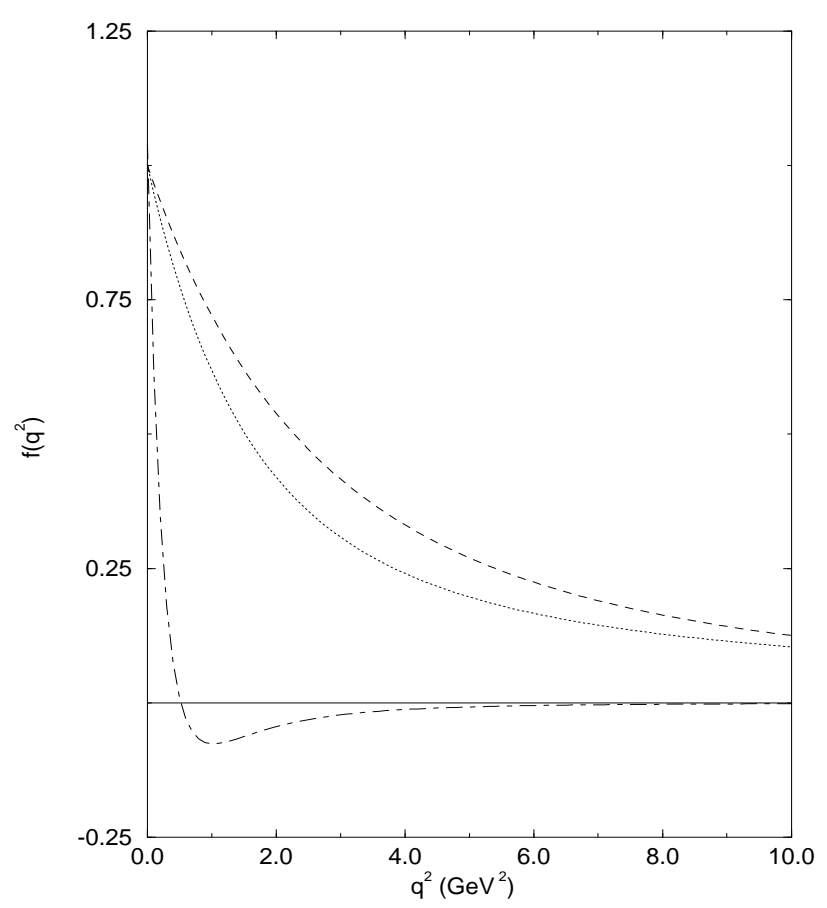

FIG. 6. Normalized elementary (parton-parton) scattering amplitudes, Eq. (9), from lattice parametrizations with the divergent term (dotted), without the divergent term (dashed), and from the Krämer-Dosch ansatz (dot-dashed).

From Fig. 14, the eikonal phase $\epsilon=\epsilon_{\mathrm{I}}+\epsilon_{\mathrm{II}}$ from the recent lattice parametrizations (including the divergent term), has a maximum at $b=0$ and decreases smoothly as the impact parameter increases. Differently, the eikonal phase from the Krämer-Dosch ansatz presents the maximum at $b \approx 0.5 \mathrm{fm}$ and reaches zero at $b=0$. These distinct behaviors come from the differences between the correlators, shown in Fig. 1.

Due to the structure of Eq. (3), the corresponding profiles present similar behaviors, as shown in Fig. 5. In this case we investigate the effect of the divergent term, referred to before. We see that, with or without the divergent term $1 /|z|^{4}$ in the correlators $D$ and $D_{1}$, the profiles present maxima at $b=0$ and a smooth decrease with the impact parameter. With and without the divergence, the profiles reach $\sim 10 \%$ of there maxima at $b=0.1$ and 0.2 $\mathrm{fm}$, respectively. We conclude that the term $1 /|z|^{4}$ does not significantly alter the outputs leading only to a more concentrated and smaller profile near $b=0$.

As a consequence of the profiles, the corresponding scattering amplitudes also present analogous similarities and differences, as can be seen in Fig. 6. Normalization of the amplitudes to 1 at $q^{2}=0$, shows that both lattice amplitudes (with and without the divergent term), present a similar smooth decrease as the momentum transfer increases, going to zero through positive values. The effect of the divergent term is a faster decrease only. Differently, the amplitude from the Krämer-Dosch ansatz decreases still faster, presenting a change of sign (zero) at $q^{2} \sim 0.5 \mathrm{GeV}^{2}$ and goes asymptotically to zero through negative values. We see that according to our calcula- tions, there is no agreement at all between profiles or amplitudes from lattice parametrizations and from the Krämer-Dosh ansatz.

\section{B. Phenomenological and semiempirical informations}

Presently, some limited informations concerning elementary profiles and/or amplitudes are available from phenomelogical models and semiempirical analysis (model independent) as explained in what follows. The usual framework of analysis is the eikonal approximation in which the elastic hadronic amplitude $F$ is related to the eikonal function $\chi$ by [19]

$$
F(q, s)=i \int b d b J_{0}(q b)\{1-\exp [i \chi(b, s)]\},
$$

where $\sqrt{s}$ is the center-of-mass energy. The hadronic amplitude is connected with physical observables as differential, total cross sections, etc., and the eikonal is defined in the context of each phenomenological model. In the simplest approach, represented by the Glauber or ChouYang formalism, the eikonal is expressed by [1, 20,21]

$$
\chi(b, s)=C \int q d q J_{0}(q b) G_{A} G_{B} f,
$$

where $G_{A}, G_{B}$ are hadronic form factors, $f$ the elementary (parton-parton) amplitude, and $C$ depends only on the energy.

Phenomenology. In the absence of theoretical predictions for both form factors and elementary amplitudes, models are yet characterized by different choices of parametrizations for these functions. As reviewed in Ref. [1], some models assume parametrizations for the electromagnetic form factors and others introduce energy -dependent form factors. With quite different choices for the elementary amplitudes all these models present satisfactory descriptions of the experimental data. For this reason it is difficult to identify a "phenomenological amplitude" since it depends on the parametrizations used for the form factors and some other specific aspects of each model. So this puts serious limitations in attempts to compare theoretical - phenomenological results and we will return to this point in the next section.

However, even taking account of these limitations, it may be useful to see what kind of similarities could be estabilished. In this sense, based on the review of Ref. [1] we could say the following.

(a) The behavior of both $\gamma(b)$ and $f(q)$ predicted from lattice parametrizations (Figs. 5 and 6) are in qualitative agreement with the form introduced by Glauber and Velasco 22]

$$
f_{\mathrm{GV}}(q, s)=\frac{1}{\sqrt{1+\frac{q^{2}}{\alpha^{2}(s)}}},
$$


where $\alpha^{2}=7.14 \mathrm{GeV}^{2}$ for $p p$ scattering at $\sqrt{s}=23$ $\mathrm{GeV}$ (with a phase factor in $f_{\mathrm{GV}}$ ) and $\alpha^{2}=0.625 \mathrm{GeV}^{2}$ for $\bar{p} p$ at $546 \mathrm{GeV}$. The elementary amplitude decreases smoothly through positive values and goes asymptotically to zero, as shown in Fig. I7, for comparison with the results from lattice parametrizations.

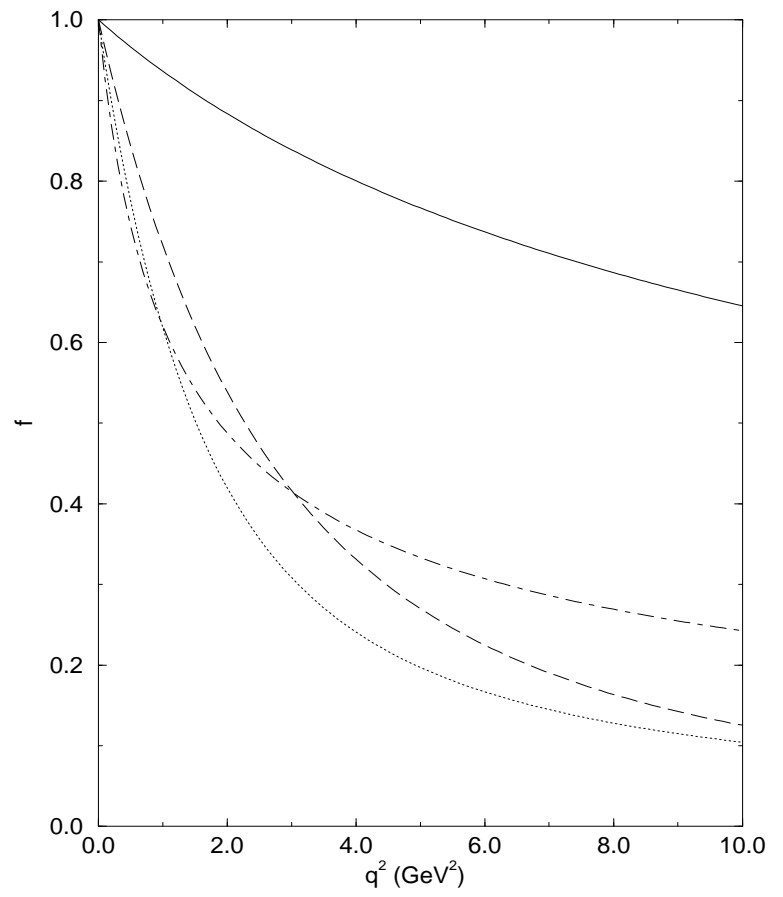

FIG. 7. Elementary amplitudes from lattice parametrizations, with (dotted) and without (dashed) the divergent terms (same as Fig. 6), compared with the Glauber and Velasco parametrization, Eq. (27), for $p p$ at $23 \mathrm{GeV}$ (solid) and $\bar{p} p$ at $546 \mathrm{GeV}$ (dot-dashed).

(b) The profiles and amplitudes from the Krämer and Dosch ansatz (Figs. 5 and 6) show qualitative agreement with the parametrizations used by Menon and Pimentel 23]:

$$
f_{\mathrm{mBSW}}=\frac{1-q^{2} / \alpha^{2}}{1+q^{4} / \alpha^{4}},
$$

where $\alpha^{2}=8.2 \mathrm{GeV}^{2}$, independent of the energy or reaction, and the subscript means modified BSW (Bourrely, Soffer, and $\mathrm{Wu}$ ), as explained in Ref. [1]. The amplitude presents a change of sign and then goes asymptotically to zero through negative values.

Semiempirical analyses. This last behavior is also predicted in the semiempirical analysis by Buenerd, Furget, and Valin [2]. These authors used the fit to $p p$ differential cross section data introduced by Amaldi and Schubert [24] and calculated the eikonal in the transfer momentum space. In the context of the Glauber-Chou-Yang model, Eq. (26), this quantity reads

$$
\chi(q, s)=C G_{p}^{2} f .
$$

The elementary amplitude $f(q)$ was then extracted at $\sqrt{s}=23.5 \mathrm{GeV}$ by dividing $\chi(q, s)$ by parametrizations for the electromagnetic form factor (Felst parametrization and a dipole form used in the Bourrely-Soffer-Wu model [2]) and normalizing the result at $q^{2}=0$ to one. The amplitude so extracted presents a change of sign at $q_{0}^{2}=8.6 \mathrm{GeV}^{2}$, going to zero through negative values. In the CERN Intersecting Storage Ring (ISR) energy region $(23.5 \mathrm{GeV} \leq \sqrt{s} \leq 62.5 \mathrm{GeV})$ the position of the zero decreases as the energy increases, reaching $q_{0}^{2}=5.0 \mathrm{GeV}^{2}$ at $\sqrt{s}=62.5 \mathrm{GeV}$ [2].

However, this kind of procedure has two critical aspects.

(1) Since experimental data are available only in a limited interval of momentum transfer $\left(q^{2} \leq 6.0 \mathrm{GeV}^{2}\right.$ in the above case) all kinds of extrapolations in the fits, allowed statistically, should be taken into account. This can be made by error propagation from the fits parameters, which, however, was not done in the above analysis.

(2) The result for the amplitude yet depends on parametrizations for the form factor. The choice to use an electromagnetic form factor is an approximation since we are treating a hadronic and not an electromagnetic interaction. However, there is no theoretical or experimental information on hadronic matter form factors.

Recently, through fit procedures to $p p$ differential cross section data, Carvalho and Menon obtained the eikonal in the transfer momentum space, taking account of error propagation and also the effect of large momentum data 25]. The result shows statistical evidence for a change of sign in the eikonal in the interval $5 \mathrm{GeV}^{2} \lesssim q^{2} \lesssim 9 \mathrm{GeV}^{2}$ at the ISR energy region [26] and asymptotical limit to zero through negative values. However, the movement of the zero with the energy cannot be inferred on statistical grounds. The analysis was performed through both a fit method and a numerical method. The former approach gives an average position of the zero at $q_{0}^{2}=7.1 \pm 0.7 \mathrm{GeV}^{2}$ and the later at $q_{0}^{2}=6.1 \pm 0.7 \mathrm{GeV}^{2}$ 25. If, in the context of Eq. (29), this zero is not associated with an hadronic form factor the result favors the amplitude from the Krämer-Dosch ansatz. Moreover, we found that the above positions of the zero are well reproduced if the gluonic correlation length in the KrämerDosch amplitude has the value $a \simeq 0.1 \mathrm{fm}$. Specificaly, $a=0.0957 \mathrm{fm}$ for $q_{0}^{2}=7.1 \mathrm{GeV}^{2}$ and $a=0.1040 \mathrm{fm}$ for $q_{0}^{2}=6.1 \mathrm{GeV}^{2}$. The result, in the case of the zero at $q_{0}^{2}=7.0 \mathrm{GeV}^{2}$, is shown in Fig. 8, together with the parametrization used by Menon and Pimentel for $\alpha^{2}=7.0 \mathrm{GeV}^{2}$ in Eq. (28).

\section{Theoretical and phenomenological issues}

The physical picture in the Nachtmann approach is that of interaction of two long-lifetime partons with a common nonperturbative QCD vacuum, the partons traveling essentially on straight lightlike world lines. It is also assumed that the transversal momenta of the partons 
(related to the hadron's movement) are orders of magnitude smaller then the forward momenta. Therefore, this picture is characterized by very small momentum transfer and asymptotically high-energy regime. These are crucial aspects of the nonperturbative approach: They specify detailed frontiers, beyond which physical interpretations and/or comparisons with phenomenological information have no justification and this demands further discussion. In Figs. 5 and 6, we display the results for the elementary profiles and amplitudes in a wide interval of impact parameter and momentum transfer. Based on the range of validity of the theoretical framework, we must see part of these curves as extrapolations to large momentum transfer and an essentially small impact parameter. Also, the elementary profiles and amplitudes do not depend on the energy since they are associated with the asymptotically high-energy limit. This leads to the following considerations.

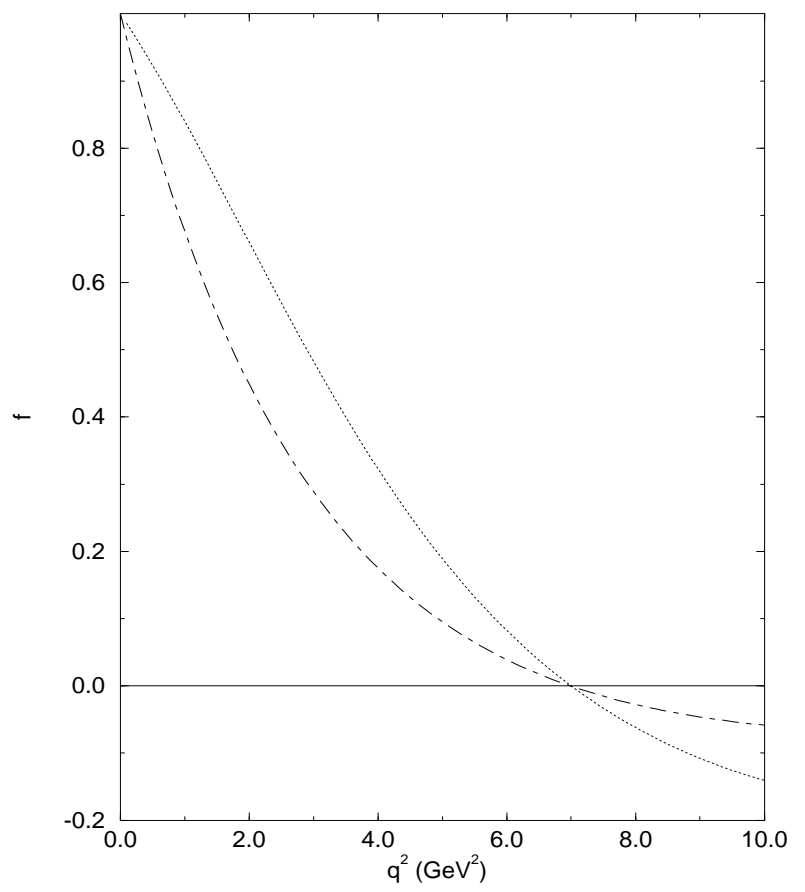

FIG. 8. Elementary amplitudes from Krämer-Dosch ansatz with correlation lenght $a=0.0957 \mathrm{fm}$ (dot-dashed) compared with the parametrization used by Menon and Pimentel, Eq. (28), with $\alpha^{2}=7.0 \mathrm{GeV}^{2}$ (dotted).

For the amplitudes obtained from the lattice parametrizations, the monotonical decrease to zero through positive values is clearly an extrapolation, at large transfer momenta. This behavior, however, could be broken, for example, by a specific model for that region, introducting scale properties, and even zeros for large $q^{2}$.

The comparison displayed in Fig. 7 between the lattice inspired amplitude and the Glauber-Velasco parametrization has also only a qualitative character, since in the last case the amplitude depends on the energy (and reaction). We see, however, a tendency to agreement as the energy increases, in the small transfer momentum region.

The general agreement between the behaviors of the Krämer-Dosch amplitude and $f_{\text {mBSw }}$ (Fig. 8) may have some more profound (but limited) meaning, since both amplitudes do not depend on the energy. Also, the connections with experimental data in the approaches of Refs. 10] and [23] are obtained with energy dependences in the hadron's transverse wave function and hadronic form factors, respectively. This is very similar from a formal point of view: The energy dependence is ultimately associated with the hadronic radius. However, the limits in the transfer momentum region must be taken into account.

Another theoretical limitation that should be stressed concerns the correlation functions determined on the lattice. As referred to in Sec. IV A the theoretical results were obtained in the finite interval $0.1-1.0 \mathrm{fm}$ and in this paper we used the parametrizations introduced in Ref. [12. With this assumption we found that the divergent term $1 /|z|^{4}$ does not affect the profile or amplitudes in a substantial way (Figs. 5, 6). Now, what kind of information can we extract from the "unknown" region 0-0.1 fm? We observed that, neglecting the divergent term in the correlation function $D(z)$ by taking $B=0$ in Eq. (13), leads to a similar result as that obtained in the early calculation, Eq. (10). Specifically, in the former case the finite $D$ value at $|z|=0$ is $\approx 4.7 \mathrm{GeV}^{4}$ and in the latter case, $\approx 5.0 \mathrm{GeV}^{4}$. From Fig. 1 and Sec. III B we see that these values are yet near three times the finite value of the Krämer-Dosch correlator $\left(\approx 1.8 \mathrm{GeV}^{4}\right)$. This difference between the lattice extrapolations (without divergence) and Krämer-Dosch ansatz in the unacessible region of small distances (0.0-0.1 fm) has strong consequences, leading to the differences observed in the corresponding profiles (Fig. 5) and amplitudes (Fig. 6). That is, the existence of zero in the amplitude seems to be associated with the particular form of the correlator at $0<|z| \lesssim 0.1 \mathrm{fm}$ (for example, exponential, Gaussian, etc.), or even at $0.1 \leq|z|<0.4 \mathrm{fm}$, and also to the value of the maximum at $|z|=0$. This small distance region, however, is not presently acessible to lattice calculations.

The results for the profiles and amplitudes showed a weaker dependence on the divergence of the type $|z|^{-4}$ than should be expected. Theoretically this kind of divergence may be associated either to operator product expansion (OPE) divergences or some specific high-energy gluon exchange process.

Our comparative analysis also brings some information on the gluonic correlation lenght $a$ that should be discussed. This quantity is a very important parameter in the SVM applied to high-energy scattering. All the formalism is strongly sensitive to its value since, for example, total cross sections for parton-parton scattering is proportional to $a^{10}$ [5]. As explained in Sec. III B, the Krämer-Dosch (KD) correlator function was determined by fit to early lattice results, from which they obtained $a=0.35 \mathrm{fm}$ and this value may reproduce parton-parton 
total cross sections of the order of $4 \mathrm{mb}$ [5]. On the other hand, from $\mathrm{SU}(2)$ lattice gauge theory, it was estimated $a=0.1 \sim 0.2 \mathrm{fm}$ [27], and both early and recent parametrizations of the correlation functions from the lattice, Eqs. (10), (13), and (14), lead to $a=0.22 \mathrm{fm}$. In the context of the SVM, $a \sim 0.2 \mathrm{fm}$ means a very small contribution to parton-parton scattering in case of using $\mathrm{KD}$ ansatz, as discussed in [5].

Concerning these distinct estimates, we showed in our analysis that for $a=0.35 \mathrm{fm}$ the elementary amplitude for the Krämer-Dosch correlator has a zero at $q^{2} \simeq 0.5 \mathrm{GeV}^{2}$ and that for $a \simeq 0.1 \mathrm{fm}$ the position changes to $q^{2}=7.0 \mathrm{GeV}^{2}$. Even taking account of the limitations related to the semiempirical analysis (Sec. $\mathrm{VB}$ ), it is evident the existence of zeros at the ISR energy region, in the interval $5.0 \leq q^{2} \leq 9.0 \mathrm{GeV}^{2}$ [2,26]. We conclude that, although this interval corresponds to finite energies, it seems to favor smaller values for the correlation lenght than $0.35 \mathrm{fm}$.

At last it should also be stressed that the lattice results we used were obtained in the quenched approximation and therefore additional uncertainties may be considered. Recently, dynamical fermions have been taken into account in lattice calculations, allowing a full QCD treatment 28]. However, since a reanalysis is in course, with improved statistics [29], we will leave this subject for future consideration. It will also be suitable to compare these new results with those presented here.

\section{FINAL CONCLUSIONS}

In the limit of extremely high energies (quarks on the light-cone) and small momentum transfer between partons, the nonperturbative approach connects the correlation functions with the parton-parton amplitudes. Making use of recent lattice parametrizations and also of the Krämer-Dosch ansatz for these correlators, we calculated the corresponding elementary amplitudes in both impact parameter and transfer momentum spaces. In the case of lattice inputs the calculations were performed with and without the divergent term recently introduced.

We found that this divergence in the correlator does not substantially affect the normalized amplitudes and also that the results from lattice parametrizations and from the Krämer-Dosch ansatz do not agree. Although the lattice parametrizations are statistically consistent with the theoretical points $\left(\chi^{2} / N_{\mathrm{DF}} \sim 1.7\right.$ [12]) there is no real theoretical information in the interval $0.0-0.1$ $\mathrm{fm}$. This is a crucial point and our analysis furnished two novel results concerning possible connections between the short range behavior of the correlators and the final results for the elementary amplitudes. On the one hand, Fig. 6 shows that the divergent term $1 /|z|^{4}$ causes only a faster decrease of the amplitude as the momentum transfer increases, that is, it does not generate local minima or zeros. The amplitude coming from this particular lat- tice parametrization, with or without the divergent term, is characterized by a monotonical decrease with the momentum transfer through positive values. On the other hand, in the case where the correlator may be finite at $|z|=0$, its maximum value and also its shape at small $|z|(|z| \lesssim 0.4 \mathrm{fm})$ strongly affect the behavior of the amplitude, as shown in Fig. 6 and quantitatively discussed in Sec. VO: Zeros (change of sign) and local minima may be generated. That is, despite the present lack of theoretical information in the short range $0.0-0.1 \mathrm{fm}$, we can infer, in the context of the stochastic vacuum model, what kind of behavior should be expected for the elementary amplitude.

Attempts to compare these results with phenomenological and/or semiempirical information on the amplitudes are limited. The reason is that this information comes from an analysis at finite energies (presently available) and the amplitudes depend on particular model assumptions or specific parametrizations for the form factors.

On the other hand, as referred to in Sec. V, the distinct results for the amplitudes from the lattice and KrämerDosch ansatz show qualitative agreement with different model predictions such as Glauber-Velasco and MenonPimentel, respectively. Furthermore, semiempirical analysis could favor the Krämer-Dosch amplitude, but with a gluonic correlator length smaller than $0.35 \mathrm{fm}$.

We conclude that, even taking account of all the limitations referred to (Secs. $\mathrm{VB}$ and $\mathrm{VO}$ ), these agreements suggest possible links between theory and phenomenological or semiempirical analyses. Further investigations along these lines may be important as a source of feedback for theoretical (nonperturbative) ideas, meanly concerning suitable considerations on energy dependences and small distance phenomena.

\section{ACKNOWLEDGMENTS}

We are grateful to Professor A. Di Giacomo for a critical reading of the manuscript and valuable discussions and suggestions. We give thanks to Capes and $\mathrm{CNPq}$ for financial support.

[1] M. J. Menon, Phys. Rev. D 48, 2007 (1993); A. F. Martini, M. J. Menon, and D. S. Thober, ibid. 54, 2385 (1996); in Frontiers in Strong Interactions, Proceedings of the VIth Blois Workshop on Elastic and Diffractive Scattering, edited by P. Chiappetta, M. Haguenauer, and J. Trân Thanh Vân (Editions Frontiéres, Gif-sur-Yvette, 1996) p. 121.

[2] C. Furget, M. Buenerd, and P. Valin, Z. Phys. C 47, 377 (1990). 
[3] P. V. Landshoff and O. Nachtmann, Z. Phys. C 35, 405 (1987).

[4] O. Nachtmann, Ann. Phys. (N.Y.) 209, 436 (1991).

[5] A. Krämer and H. G. Dosch, Phys. Lett. B 252, 669 (1990).

[6] H. G. Dosch, Phys. Lett. B 190, 177 (1987).

[7] H. G. Dosch and Yu. A. Simonov, Phys. Lett. B 205, 339 (1988).

[8] N. G. van Kampen, Physica (Amsterdam) 74, 215 (1974).

[9] Yu. A. Simonov, Nucl. Phys. B204, 67 (1989).

[10] H. G. Dosch, E. Ferreira, and A. Krämer, Phys. Rev. D 50, 1992 (1994).

[11] A. Di Giacomo and H. Panagopoulos, Phys. Lett. B 285, 133 (1992).

[12] A. Di Giacomo, E. Meggiolaro, and H. Panagopoulos, Nucl. Phys. B483, 371 (1997).

[13] U. Grandel and W. Weise, Phys. Lett. B 356, 567 (1995).

[14] M. Campostrini, A. Di Giacomo, M. Maggiore, H. Panagopoulos, and E. Vicari, Phys. Lett. B 225, 403 (1989).

[15] A. Di Giacomo, M. Maggiore, and S. Olejnik, Phys. Lett. B 236, 199 (1990); Nucl. Phys. B347, 441 (1990).

[16] C. Michael and M. Teper, Nucl. Phys. B305, 453 (1988).

[17] NAG FORTRAN Library Manual, Mark 16, 1993; S. Wolfram, MATHEMATICA (Addison-Wesley, New York, 1991).

[18] F. James and M. Roos, MINUIT-Function Minimization and Error Analysis, CERN Report No. D506 (CERN, Geneva, 1992).

[19] R. J. Glauber, in Lectures in Theoretical Physics, edited by W. E. Britten et al. (Interscience, New York, 1959), Vol. I, p. 315.

[20] W. Czyż and L. C. Maximon, Ann. Phys. (N.Y.) 52, 59 (1969); V. Franco and G. K. Varma, Phys. Rev. C 18, 349 (1978).

[21] T.T. Chou and C.N. Yang, Phys. Rev. 175, 1832 (1968).

[22] R.J. Glauber and J. Velasco, Phys. Lett. B 147, 380 (1984); in Proceedings of the Second International Conference on Elastic and Diffractive Scattering, New York, 1987, edited by K. Goulianos (Editions Frontières, Gifsur-Yvette, 1988), p. 219.

[23] M.J. Menon and B.M. Pimentel, Hadronic J. 13, 325 (1990); M.J. Menon, in Proceedings of the Fourth International Conference on Elastic and Diffractive Scattering, Elba, Italy, 1991, edited by P. Cervelli and S. Zucchelli [Nucl. Phys. B (Proc. Suppl.) 25, 94 (1992)]; Canadian J. Phys. 74, 594 (1996).

[24] U. Amaldi and K.R. Schubert, Nucl. Phys. B166, 301 (1980).

[25] P. A. S. Carvalho and M. J. Menon, in VIII Workshop on Hadronic Interactions, edited by Y. Hama, F. S. Navarra, and M. Nielsen (Instituto de Física - USP, São Paulo, 1997), p. 12.

[26] P. A. S. Carvalho and M. J. Menon, Phys. Rev. D 56, 7321 (1997).

[27] M. Campostrini, A. Di Giacomo, and G. Mussardo, Z. Phys. C 25, 173 (1984).

[28] A. Di Giacomo, E. Meggiolaro, and H. Panagopoulos, Phys. Lett. B 408, 315 (1997).
[29] A. Di Giacomo (private communication). 